\title{
Jet Substructure Measurements Sensitive to Soft QCD ef- fects with the ATLAS Detector
}

\author{
Gabriele Chiodini ${ }^{1, *}$ On behalf of the ATLAS collaboration \\ ${ }^{1}$ Istituto Nazionale di Fisica Nucleare, sezione di Lecce, Italia
}

\begin{abstract}
Jet substructure observables are powerful tools for the identification of boosted heavy particles and for probing QCD at different energy scales in proton-proton collisions at the LHC. In this work three recent ATLAS measurements sensitive to soft QCD effects and performed with the use of jet substructures are described. Their are $k_{t}$ splitting scales, soft-drop jet masses and pull-jet angles. Some discrepancies between data and predictions are observed in the non-perturbative regime.
\end{abstract}

\section{Introduction}

The features of energy flow within the body of hadronic jets are very useful for probing perturbative and non-perturbative QCD at hadron colliders. Nowadays, their analysis is used in many studies.

Jet substructure observables are very relevant for an efficient identification of hadronically decaying boosted objects, such as $\mathrm{W}, \mathrm{Z}, \mathrm{H}$ and t, extending significantly the probed energy range and discovery capability of LHC experiments such as ATLAS [1]. They are measured in data, unfolded for detector effects using an iterative Bayesian technique [2], and compared to QCD calculations and particle-level MC simulations.

\section{Sequential clustering algorithm}

Jets are reconstructed from a set of particle momenta (reconstructed from calorimeter energy deposits, charged tracks, simulated particles) which are close in the rapidity-azimuthal $y-\phi$ plane according to a clustering algorithm.

At the LHC sequential clustering algorithms [3] are used to reconstruct jets. They have the nice property to be infrared- and collinear-safe, allowing a meaningful comparison between data and theoretical predictions.

The algorithms iteratively combine the nearest pair of jet constituent $\mathrm{j}_{i}$ and $\mathrm{j}_{j}$, in the $\mathrm{y}-\phi$ plane, according to a distance $\mathrm{d}_{i j}$ computed as the geometrical distance $\Delta R_{i j}^{2}=\sqrt{\Delta y_{i j}^{2}+\Delta \phi_{i j}^{2}}$ normalized to the square of the radius parameter $\mathrm{R}$ adopted and weighted by the minimum of a power $\mathrm{n}$ of the transverse momenta and defined by the formula:

$$
d_{i j}=\min \left(p_{T, i}^{n}, p_{T, j}^{n}\right) \frac{\Delta R_{i j}^{2}}{R^{2}},
$$

*e-mail: gabriele.chiodini@le.infn.it 
where the value of $\mathrm{R}$ is usually chosen between 0.2 and 1 . If the minimum distance at the $\mathrm{k}$-th step $\mathrm{d}_{k}=\min _{i, j}\left\{d_{i j}\right\}$ is larger than $d_{i b}=p_{T, i}^{n}$ then the jet constituent $\mathrm{j}_{i}$ is promoted to a jet and removed from the jet constituent list. The clustering algorithm is repeated again on the list until all jet constituents are clustered in a jet.

The value of the exponent $\mathrm{n}$ sets for specific clustering algorithm used:

- $\mathrm{n}=2$ corresponds to the $k_{t}$ algorithm where close and soft constituents pairs are clustered first, miming the infrared and collinear splittings.

- $\mathrm{n}=-2$ corresponds to the anti- $k_{t}$ algorithm where close and hard constituents are clustered first, producing regularly shaped jets in $\mathrm{y}-\phi$ space.

- $\mathrm{n}=0$ corresponds to the Cambridge-Aachen (CA) algorithm $\left(d_{i j}=\frac{\Delta R_{i j}^{2}}{R^{2}}\right.$ and $\left.d_{i b}=0\right)$ where the geometrically closest constituent pairs are clustered first, miming the angular-ordered parton shower (PS).

If one is interested in studying jets, the anti- $k_{t}$ algorithm is the best choice because it is only weakly susceptible to the underlying event and pile-up. However, if one is interested in jet substructures, only the $k_{t}$ and CA algorithms are appropriate. This is clearly seen at the second-last stage of the clustering sequence where the last two sub-jets can reveal a hard underlying structure for the $k_{t}$ and CA algorithms but not for the anti- $k_{t}$ algorithm. As shown in the next sections, jet substructure observables are built by following the steps of a sequential clustering algorithm in reverse order.

\section{Measurements of $k_{t}$ splitting scales}

A measurement of the splitting scales of the $k_{t}$ jet-clustering algorithm was done with the ATLAS detector, in p-p collisions at $8 \mathrm{TeV}$ center-of-mass energy and $20.2 \mathrm{fb}^{-1}$ integrated luminosity, for jets produced in association with a $\mathrm{Z}$ boson decaying leptonically $(\mathrm{p}+\mathrm{p} \rightarrow \mathrm{Z}(11)+\mathrm{jets})$ and reconstructed with charged tracks (track-jets) [4].

The $\mathrm{k}$-th order splitting scale is defined by $\sqrt{d_{k}}$ for $\mathrm{n}=2$ (see previous section) with the number $\mathrm{k}$ indexing the $k_{t}$ clustering sequence in reverse order. The 0 -th order splitting scale corresponds to the final jet transverse momentum $\sqrt{d_{0}}=p_{T}$, the 1-st order splitting scale corresponds to $\sqrt{d_{1}}=\min \left(p_{T, 1}, p_{T, 2}\right) \frac{\Delta R_{12}}{R}$ of the penultimate stage of the clustering, and so on for all other orders.

Figure 1 shows two examples of splitting scale distribution measured for events with $\mathrm{Z}$ decaying in the muon and electron channel and jet radius 0.4 and 1, respectively. In reference [4] measurements up to 7-th order splitting scales are reported. Large discrepancy between data and predictions are observed at low values of the splitting scale. It is relevant to note that soft and collinear splitting have low values of $\sqrt{d_{k}}$, instead the hard splitting has high values.

\section{Measurements of soft-drop jet mass}

The calculation beyond leading log (LL) accuracy for observable related to jets is problematic due to non-global logarithms (NGLs) related to particles that radiate out of, and then radiate back into, a jet [5]. The so-called soft-drop grooming algorithm makes jet substructure insensitive to NGLs terms because it removes energy in jet related to soft QCD processes and pile-up [6]. The algorithm starts by re-clustering the jet's constituents with the CA algorithm and removes sub-jet pairs from the clustering history sequence if they do not satisfy the following criteria: 

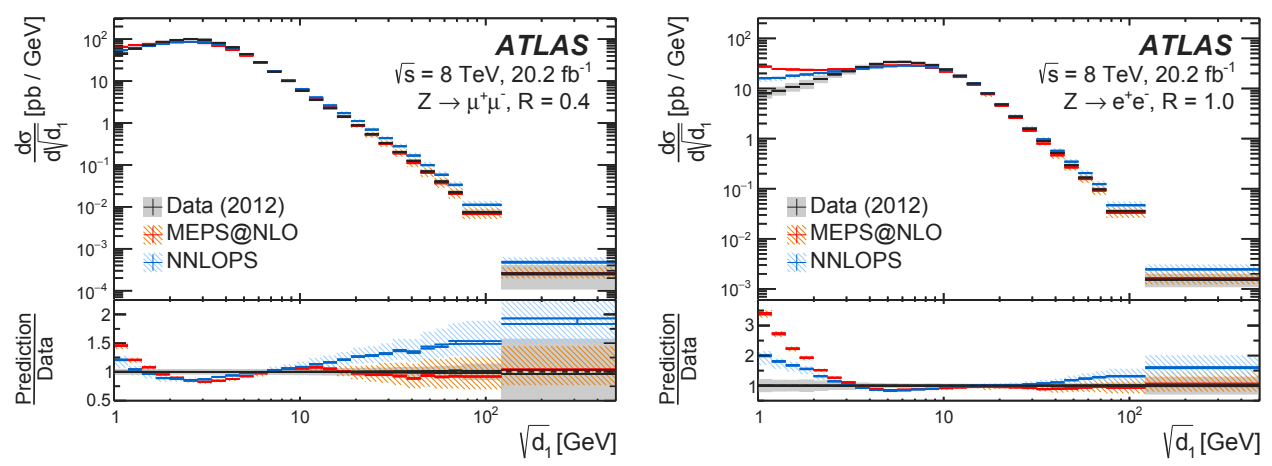

Figure 1. Left plot: charged (measured) distribution for the 1-st order splitting scales in the muon channel using the jet-radius parameter $\mathrm{R}=0.4$. Right plot: charged (measured) + neutral (extrapolated) distribution for the 1-st order splitting scales in the electron channel using the jet-radius parameter $\mathrm{R}$ $=1$. The size of the error bars reflects the statistical uncertainty, while the combined statistical and systematic uncertainty is indicated by the grey band. Theoretical predictions from Sherpa with NLO multijet merging ("MEPS@NLO") and from POWHEG+PYTHIA 8 with NNLO matching ("NNLOPS") are displayed including error bands for the generator uncertainties [4].

$$
\frac{\min \left(p_{T, 1}, p_{T, 2}\right)}{p_{T, 1}+p_{T, 2}}>z_{\text {cut }}\left(\frac{\Delta R_{12}}{R}\right)^{\beta}
$$

where the parameter $\mathbf{z}_{c u t}$ controls how much energy is removed from the jet and $\beta$ tunes the sensitivity of the algorithm to wide-angle radiation.

A very important application of the soft-drop is the measurements of the jet mass which is a powerful tool for identifying boosted hadronically decaying massive particles [7]. In fact, the jet mass of soft-drop groomed jet is insensitive to NGLs terms and it has been calculated at both next-to-leading order (NLO) with next-to-leading-logarithm NLL and leading order (LO) with next-to-next-to-leading-logarithm (NNLL) accuracy.

The soft-drop jet mass $\mathrm{m}^{\text {soft-drop }}$ of the two leading anti- $k_{t}$ jets in di-jet events $(\mathrm{p}+\mathrm{p} \rightarrow \mathrm{jet}+\mathrm{jet})$ was measured with the ATLAS detector, in $\mathrm{p}-\mathrm{p}$ collisions at $13 \mathrm{TeV}$ centerof-mass energy and $32.9 \mathrm{fb}^{-1}$ integrated luminosity [8]. The soft-drop jet mass is measured relative to the jet transverse momentum before grooming in terms of the dimensionless ratio $\rho=\mathrm{m}^{\text {soft-drop }} / p_{T}^{\text {ungroomed }}$.

The experimental distributions of $\log _{10} \rho^{2}$ is compared with several predictions and two of them are reported in Fig.2. The main focus is on the region $-3.7<\log _{10} \rho^{2}<-1.7$ of the recent precision calculations, where resummation dominates over non-perturbative $-3.7<\log _{10} \rho^{2}$ or fixed-order $\log _{10} \rho^{2}<-1.7$ regions. Studying the distribution in $\log$-scale allows the resummation region to be studied more closely. In regions where non-perturbative effects are expected to be significant, the calculations disagree with the data and a better description is provided by MC simulation.

\section{Measurements of color flow}

Despite the strong effort in the past to measure the potential effects of colour connections between partons sharing color flow, where more radiation is expected from colour coherence effects [9], it remains a poorly constrained effect of QCD and further experimental input are clearly welcome. 

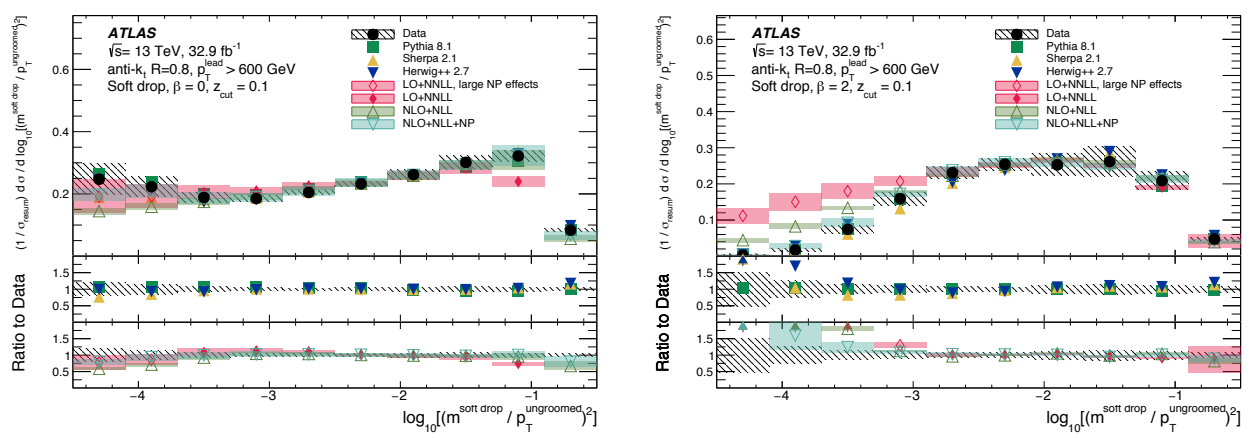

Figure 2. The unfolded $\log _{10} \rho^{2}$ distribution for anti- $k_{t}$ jets of radius $\mathrm{R}=0.8$ with $\mathrm{p}_{T}^{\text {leading }}>600 \mathrm{GeV}$, after the soft-drop algorithm is applied for $\beta=0$ (left plot) and $\beta=2$ (right plot) in data compared to PythIA, SHERPA, and HERWIG++ particle-level, and NLO+NLL+NP and LO+NNLL theory predictions. The LO+NNLL calculation does not have non-perturbative (NP) corrections; the region where these are expected to be large is shown in a open marker, while regions where they are expected to be small are shown with a filled marker. All uncertainties described in the text are shown on the data; the uncertainties from the calculations are shown on each one. The distributions are normalized to the integrated cross section measured in the resummation region [8].

In the production of a t't pair four colour-charged final state particles can be identified: the two b-quarks produced directly by the decay of the top-quarks and the two quarks produced by the hadronically decaying $\mathrm{W}$ boson. As the $\mathrm{W}$ boson does not carry colour charge the two daughter quarks must share a colour flow. The two b-quarks carry the colour charge of their respective top-quark parents, and are thus not expected to be colour connected (see Fig.3 left).

The color connection between two jets $\mathrm{j}_{1}$ and $\mathrm{j}_{2}$ (see Fig. 3 right) can be studied using the jet-pull angle $\theta_{\mathcal{P}}$ defined as the angle between the jet-pull vector $\mathcal{P}\left(\vec{j}_{1}\right)$ and the vector difference in the $y-\phi$ plane of $j_{1}$ and $j_{2}$ (jet-connection vector). The jet-pull vector of $j_{1}$ defined by the formula:

$$
\mathcal{P}\left(\vec{j}_{1}\right)=\sum_{i \in j_{1}} \frac{\left|\overrightarrow{\Delta r}_{i}\right| p_{T}^{i}}{p_{T}^{j_{1}}} \overrightarrow{\Delta r_{i}}
$$

is a $\mathrm{p}_{T}$-weighted radial moment of the jet constituents.

Figure 4 shows the jet-pull angle measured with the ATLAS detector, in p-p collisions at $13 \mathrm{TeV}$ center-of-mass energy and $36.1 \mathrm{fb}^{-1}$ integrated luminosity [10]. Jets are reconstructed using the anti- $k_{t}$ algorithm with $\mathrm{R}=0.4$ starting from three-dimensional, massless, positiveenergy topological clusters [11] reconstructed from energy deposited in the calorimeters. Semileptonic top pair events were selected in such a way to have a jet pair from the hadronic $\mathrm{W}$ decay (color connected) of a top decay and a b-jet pair from the two top decays (not-color connected) and to trigger on the high $\mathrm{p}_{T}$ lepton from the leptonic $\mathrm{W}$ decay of the other top decay.

As expected, the jet-pull angle shows a flat distribution for pairs of jets not sharing a color flow and a distribution accumulating at 0 in case of color connection. In Fig. 4 it is evident that color connection looks stronger in predictions than in data. 

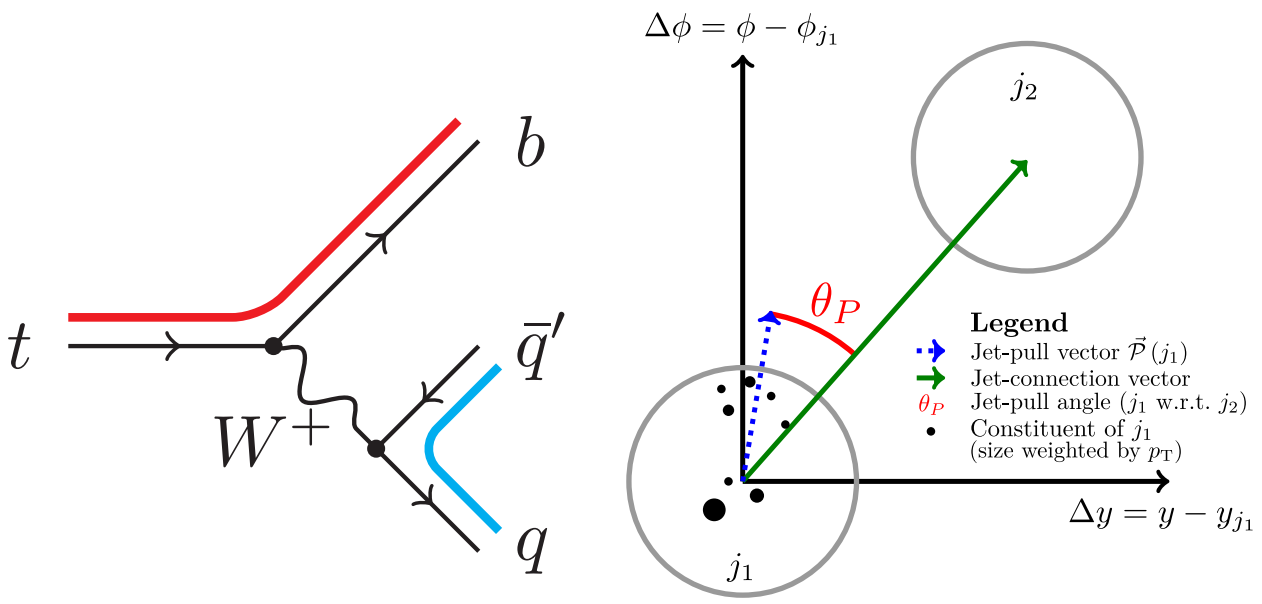

Figure 3. Left: Illustration of a hadronic top decay with typical colour connections (thick coloured lines). Right: illustration of jet-pull observables for a dijet system [10].
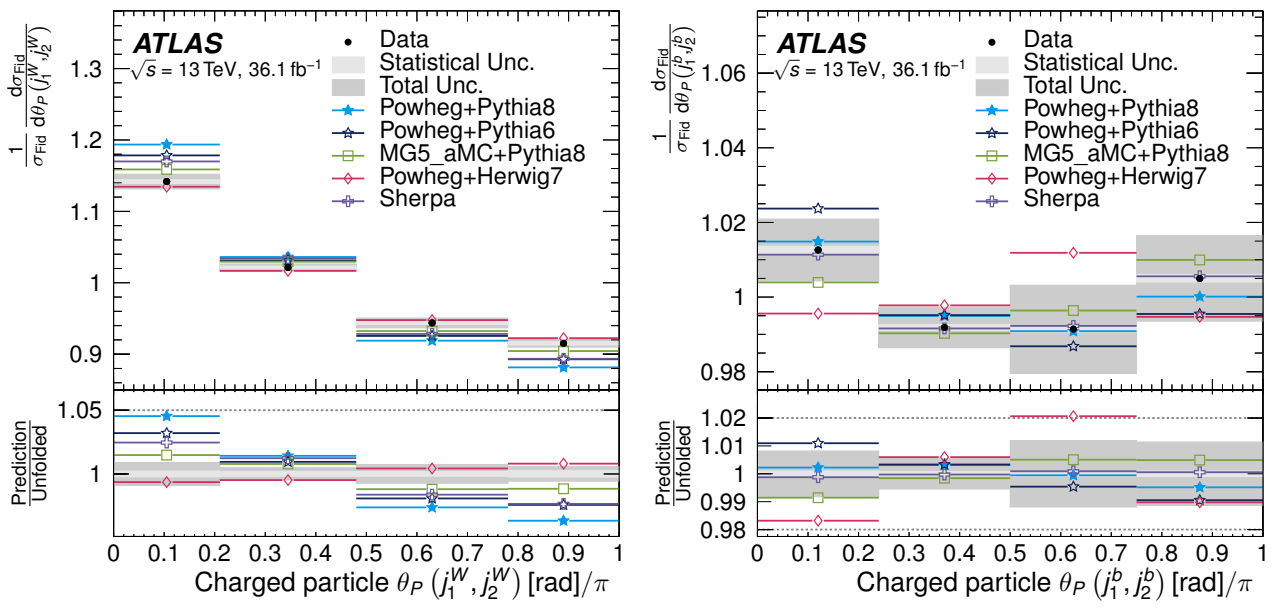

Figure 4. Normalised fiducial differential cross-sections as a function of the forward pull angle for the hadronically decaying $\mathrm{W}$ boson daughters (left plot) and for the di-b-jet (right plot) in top pair events. The forward pull angle corresponds to the pull vector of the leading $\mathrm{p}_{T}$ jet and connection vector to the sub-leading $\mathrm{p}_{T}$ jet. The data are compared to various predictions. The statistical uncertainties in the predictions are smaller than the marker size [10].

\section{Conclusions}

The reported ATLAS measurements of soft QCD effects at the LHC constrain analytical calculations in the perturbative regime. The discrepancy between predictions and data observed in the non-perturbative regime are helpful to tune $\mathrm{MC}$ in this regime, which is sensitive to pile-up and underlying event. 


\section{References}

[1] ATLAS Collaboration, 2008 JINST 3 S08003.

[2] G. D’Agostini, Nucl. Instrum. Meth. A 362, 487 (1995).

[3] G.P. Salam, Eur. Phys. J. C 67 637, (2010). arXiv:0906.1833 [hep-ex]. R. Atkin, J. Phys. Conf. Ser. 645012008 (2015).

[4] ATLAS Collaboration, JHEP08 026 (2017). arXiv:1704.01530 [hep-ex].

[5] A. Banfi et al., JHEP 08064 (2010). arXiv: 1004.3483 [hep-ex].

[6] M. Rubin, JHEP 05005 (2010). arXiv: 1002.4557 [hep-ex].

[7] A. Abdesselam, et al., Eur. Phys. J. C71, 1661 (2011). arXiv:1012.5412 [hep-ex].

[8] ATLAS Collaboration, Phys. Rev. Lett. 121, 092001 (2018). arXiv:1711.08341 [hep-ex].

[9] R. K. Ellis, W. J. Stirling and B. R. Webber, QCD and Collider Physics, Cambridge University Press, 2003.

[10] ATLAS Collaboration, CERN-EP-2018-041. http://cds.cern.ch/record/2316982. arXiv:1805.02935 [hep-ex]. Submitted to EPJC.

[11] ATLAS Collaboration, Eur. Phys. J. C 77 490, (2017). arXiv: 1603.02934 [hep-ex]. 